\title{
Multirate Formulation for Mismatch Sensitivity Analysis of Analog-to-Digital Converters That Utilize Parallel $\Sigma \Delta$-Modulators
}

\author{
Anton Blad, Håkan Johansson, and Per Löwenborg \\ Division of Electronics Systems, Department of Electrical Engineering, Linköping University, Sweden \\ Correspondence should be addressed to Anton Blad, antonb@isy.liu.se
}

Received 1 June 2007; Accepted 21 October 2007

Recommended by Boris Murmann

A general formulation based on multirate filterbank theory for analog-to-digital converters using parallel sigmadelta modulators in conjunction with modulation sequences is presented. The time-interleaved modulators (TIMs), Hadamard modulators (HMs), and frequency-band decomposition modulators (FBDMs) can be viewed as special cases of the proposed description. The usefulness of the formulation stems from its ability to analyze a system's sensitivity to aliasing due to channel mismatch and modulation sequence level errors. Both Nyquist-rate and oversampled systems are considered, and it is shown how the matching requirements between channels can be reduced for oversampled systems. The new formulation is useful also for the derivation of new modulation schemes, and an example is given of how it can be used in this context.

Copyright ( 2008 Anton Blad et al. This is an open access article distributed under the Creative Commons Attribution License, which permits unrestricted use, distribution, and reproduction in any medium, provided the original work is properly cited.

\section{INTRODUCTION}

Traditionally, analog-to-digital converters (ADCs) and digital-to-analog converters (DACs) based on $\Sigma \Delta$-modulation have been used primarily for low bandwidth and highresolution applications such as audio application. The requirements make the architecture perfectly suited for this purpose. However, in later years, advancements in VLSI technology have allowed greatly increased clock frequencies, and $\Sigma \Delta$-ADCs with bandwidths of tens of $\mathrm{MHz}$ have been reported $[1,2]$. This makes it possible to use $\Sigma \Delta$-ADCs in a wider context, for example, in wireless communications. One of the most attractive features of $\Sigma \Delta$-ADCs is their relaxed requirements on the analog circuitry, which is especially important in wireless communications where integration in analog-hostile deep submicron CMOS is favorable. However, the high-operating frequencies used for the realization of such wideband converters result in devices with high analog power consumption.

One way to reduce the operating frequency is to use several modulators in parallel, where a part of the input signal is converted in each channel. Several flavors of such $\Sigma \Delta$-ADCs have been proposed, and these can essentially be divided into four categories: time-interleaved modulators (TIMs) [3, 4], Hadamard modulators (HMs) [4-8], frequency-band decomposed modulators (FBDMs) $[4,9,10]$ and multirate modulators based on block-digital filtering [11-14]. In the TIM, samples are interleaved in time between the channels. Each modulator is running at the input sampling rate, with its input grounded between consecutive samples. This is a simple scheme, but as interleaving causes aliasing of the spectrum, the channels have to be carefully matched in order to cancel aliasing in the deinterleaving at the output. In an HM, the signal is modulated by a sequence constructed from the rows of a Hadamard matrix. One advantage over the TIM is an inherent coding gain, which increases the dynamic range of the ADC [4], whereas a disadvantage is that the number of channels is restricted to a number for which there exists a known Hadamard matrix. Another advantage, as will be shown in this paper, is the reduced sensitivity to mismatches in the analog circuitry. The third category of parallel modulators is the FBDM, in which the signal is decomposed in frequency rather than time. This scheme is insensitive to analog mismatches, but has increased hardware complexity because it requires the use of bandpass modulators. The idea of the multirate modulators is different, based on a polyphase 
decomposition of the integrator in one channel. Thus the architecture is not directly comparable to the systems described in this paper.

The parallel systems have been analyzed both in the timedomain and the frequency-domain [3, 4, 6-8, 12, 15-17], and in [18] an attempt was made to formulate a general model covering the TIM, HM, and FBDM systems. The formulation in this paper is slightly different from the one in [18] due to differences in the usage of causal and noncausal delays. The overall ADC was formulated in terms of circulant and pseudocirculant matrices, and the formulation is derived from multirate filter bank theory. The formulation is refined in this paper, and extended with a more comprehensive sensitivity analysis. Using the formulation, the behavior of a practical ADC with channel gain and modulation sequence level mismatches present can be analyzed, and it is apparent why some schemes are sensitive to mismatches between channels whereas others are not. Also, it is found that some schemes (in particular the HM systems) suffer from sensitivity in a limited set of channels such that "full calibration" between the channels is not needed. Whereas the new formulation is in fact not constrained to parallel $\Sigma \Delta$-ADCs but applicable to general parallel systems that use modulation sequences, it is described in that context in this paper as this application is considered to be particularly interesting. Further, the usefulness of the new formulation is not only limited to the analysis of existing schemes, but also for the derivation of new ones, which is demonstrated in the paper.

The organization of the paper is as follows. In Section 2, the multirate formulation of a parallel system is derived, and the signal input-to-output relation of the system is analyzed. Conditions for the system to be free from nonlinear distortion (i.e., free from aliasing) are stated. In Section 3, the sensitivity to channel mismatches for a system is analyzed in the context of the multirate formulation. In Section 4, the formulation is used to analyze the behavior of some representative systems, and also the derivation of a new scheme that is insensitive to some mismatches is presented. In Section 5, the quantization noise properties of a parallel system is analyzed. Finally, Section 6 concludes the paper.

\section{LINEAR SYSTEM MODEL}

We consider the scheme depicted in Figure 1. In this scheme, the input signal $x(n)$ is first divided into $N$ channels. In each channel $k, k=0,1, \ldots, N-1$, the signal is first modulated by the $M$-periodic sequence $a_{k}(n)=a_{k}(n+M)$. The resulting sequence is then fed into a $\Sigma \Delta$-modulator $\Sigma \Delta_{k}$, followed by a digital filter $G_{k}(z)$. The output of the filter is modulated by the $M$-periodic sequence $b_{k}(n)=b_{k}(n+M)$ which produces the channel output sequence $y_{k}(n)$. Finally, the overall output sequence $y(n)$ is obtained by summing all channel output sequences. The $\Sigma \Delta$-modulator in each channel works in the same way as an ordinary $\Sigma \Delta$-modulator. By increasing the channel oversampling, and reducing the passband width of the channel filter accordingly, most of the shaped noise is removed, and the resolution is increased. By using several channels in parallel, wider signal bands can be handled without increasing the input sampling rate to unreasonable

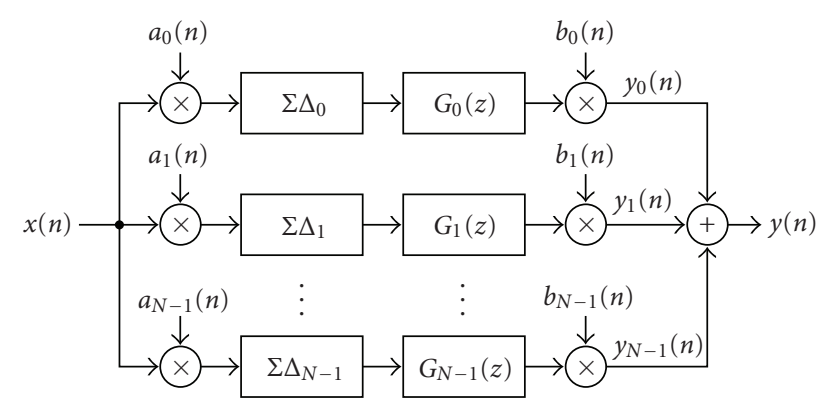

FIGURE 1: ADC system using parallel $\Sigma \Delta$-modulators and modulation sequences.

values. In other words, instead of using one single $\Sigma \Delta$-ADC with a very high input sampling rate, a number of $\Sigma \Delta$-ADCs in parallel provide essentially the same resolution but with a reasonable input sampling rate.

The overall output $y(n)$ is determined by the input $x(n)$, the signal transfer function of the system, and the quantization noise generated in the $\Sigma \Delta$-modulators. Using a linear model for analysis, the signal input-to-output relation and noise input-to-output relation can be analyzed separately. The signal transfer function from $x(n)$ to $y(n)$ should be equal to (or at least approximate) a delay in the signal frequency band of interest. The main problem in practice is that the overall scheme is subject to channel gain, offset, and modulation sequence level mismatches $[4,15,16]$. This is where the new general formulation becomes very useful as it gives a relation between the input and output from which one can easily deduce a particular scheme's sensitivity to mismatch errors. The noise contribution, on the other hand, is essentially unaffected by channel mismatches. Therefore, the noise analysis can be handled in the traditional way, as in Section 5.

\subsection{Signal transfer function}

From the signal input-to-output point of view, we have the system depicted in Figure 2(a) for channel $k$. Here, each $H_{k}(z)$ represents a cascade of the corresponding signal transfer function of the $\Sigma \Delta$-modulator and the digital filter $G_{k}(z)$. To derive a useful input-output relation in the $z$-domain, we make use of multirate filter bank theory [19]. As $a_{k}(n)$ and $b_{k}(n)$ are $M$-periodic sequences, each multiplication can be modelled as $M$ branches with constant multiplications and the samples interleaved between the branches. This is shown in the structure in Figure 2(b), where

$$
\begin{aligned}
& a_{k, n}= \begin{cases}a_{k}(0) & \text { for } n=0, \\
a_{k}(M-1) & \text { for } n=1,2, \ldots, M-1,\end{cases} \\
& b_{k, n}=b_{k}(M-1-n) \text { for } n=0,1, \ldots, M-1 .
\end{aligned}
$$

Now, consider the system shown in Figure 3, representing the path from $x_{q}(m)$ to $y_{k, r}(m)$ in Figure 2(b). Denoting

$$
\tilde{H}_{k}(z)=z^{M-1} H_{k}(z),
$$




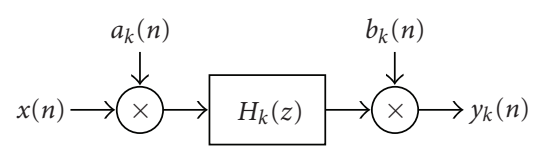

(a) Model of channel

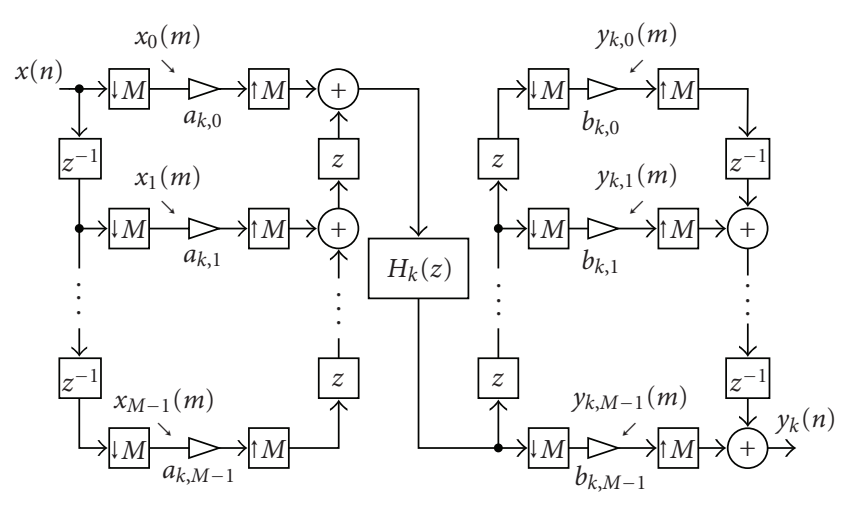

(b) Polyphase decomposition of multipliers

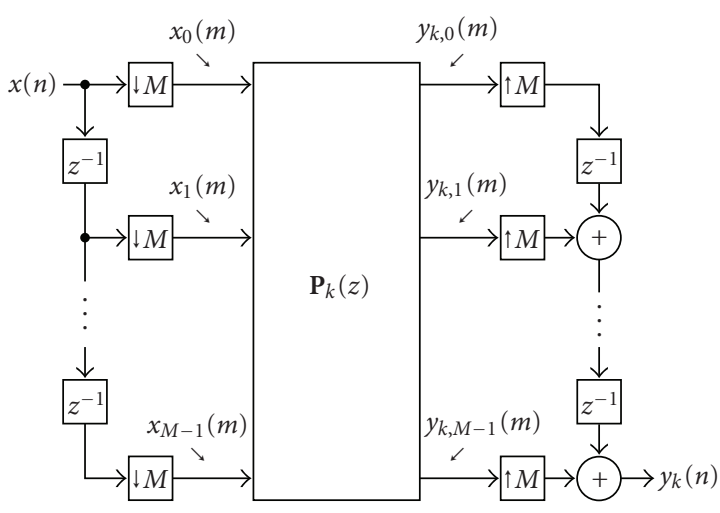

(c) Multirate formulation of a channel

FIgURE 2: Equivalent signal transfer models of a channel of the parallel system in Figure 1.

the transfer function from $x_{q}(m)$ to $y_{k, r}(m)$ is given by the first polyphase component in the polyphase decomposition of $z^{q} \tilde{H}_{k}(z) z^{-r}$, scaled by $a_{k, q} b_{k, r}$. For $p=q-r=0,1, \ldots, M-$ 1 , the polyphase decomposition of $z^{p} \tilde{H}_{k}(z)$ can be written

$$
z^{p} \tilde{H}_{k}(z)=\sum_{i=0}^{M-1} z^{p-i} \tilde{H}_{k, i}\left(z^{M}\right)
$$

and the first polyphase component is $\widetilde{H}_{k, p}(z)$, that is, the $p$ th polyphase component of $\tilde{H}_{k}(z)$ as specified by the Type 1 polyphase representation in [19]. For $p=-M+1, \ldots,-1$,

$$
z^{p} \tilde{H}_{k}(z)=\sum_{i=0}^{M-1} z^{p-i+M} z^{-M} \tilde{H}_{k, i}\left(z^{M}\right)
$$

and the first polyphase component is $z^{-1} \tilde{H}_{k, p+M}(z)$. Returning to the system in Figure 2(b), the transfer functions $P_{k}^{r, q}(z)$ from $x_{q}(m)$ to $y_{k, r}(m)$ can now be written

$$
P_{k}^{r, q}(z)= \begin{cases}b_{k, r} \tilde{H}_{k, q-r}(z) a_{k, q} & \text { for } q \geq r, \\ b_{k, r} z^{-1} \widetilde{H}_{k, q-r+M}(z) a_{k, q} & \text { for } q<r .\end{cases}
$$

The relations can be written in matrix form as $\mathbf{P}_{k}(z)$ in

$$
\mathbf{P}_{k}(z)=\left[\begin{array}{ccccc}
A_{1} & A_{5} & A_{9} & \cdots & A_{13} \\
A_{2} & A_{6} & A_{10} & \cdots & A_{14} \\
A_{3} & A_{7} & A_{11} & \cdots & A_{15} \\
\vdots & \vdots & \vdots & \ddots & \vdots \\
A_{4} & A_{8} & A_{12} & \cdots & A_{16}
\end{array}\right],
$$

where

$$
\begin{array}{lrl}
A_{1}=a_{k, 0} b_{k, 0} \tilde{H}_{k, 0}(z), & A_{2}=a_{k, 0} b_{k, 1} z^{-1} \tilde{H}_{k, M-1}(z), \\
A_{3}=a_{k, 0} b_{k, 2} z^{-1} \tilde{H}_{k, M-2}(z), & A_{4}=a_{k, 0} b_{k, M-1} z^{-1} \tilde{H}_{k, 1}(z), \\
A_{5}=a_{k, 1} b_{k, 0} \tilde{H}_{k, 1}(z), & A_{6}=a_{k, 1} b_{k, 1} \tilde{H}_{k, 0}(z), \\
A_{7}=a_{k, 1} b_{k, 2} z^{-1} \tilde{H}_{k, M-1}(z), & A_{8}=a_{k, 1} b_{k, M-1} z^{-1} \tilde{H}_{k, 2}(z), \\
A_{9}=a_{k, 2} b_{k, 0} \tilde{H}_{k, 2}(z), & A_{10}=a_{k, 2} b_{k, 1} \tilde{H}_{k, 1}(z), \\
A_{11}=a_{k, 2} b_{k, 2} \tilde{H}_{k, 0}(z), & A_{12}=a_{k, 2} b_{k, M-1} z^{-1} \tilde{H}_{k, 3}(z), \\
A_{13}=a_{k, M-1} b_{k, 0} \tilde{H}_{k, M-1}(z), & A_{14}=a_{k, M-1} b_{k, 1} \tilde{H}_{k, M-2}(z), \\
A_{15}=a_{k, M-1} b_{k, 2} \tilde{H}_{k, M-3}(z), & A_{16}=a_{k, M-1} b_{k, M-1} \tilde{H}_{k, 0}(z),
\end{array}
$$

and it is thus obvious that one channel of the system can be represented by the structure in Figure 2(c). In the whole system (Figure 1) a number of such channels are summed at the output, and the parallel system of $N$ channels can be represented by the structure in Figure 4, where the matrix $\mathbf{P}(z)$ is given by

$$
\mathbf{P}(z)=\sum_{k=0}^{N-1} \mathbf{P}_{k}(z)
$$

For convenience, we write (6) as

$$
\mathbf{P}_{k}(z)=\mathbf{S}_{k} \cdot \tilde{\mathbf{H}}_{k}(z),
$$




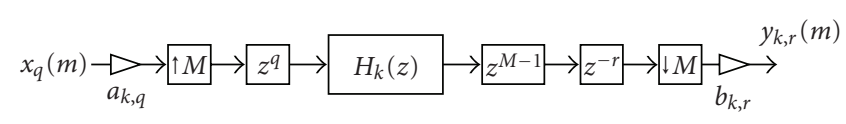

FIgURE 3: Path from $x_{q}(m)$ to $y_{k, r}(m)$ in channel $k$ as depicted in Figure 2(b).

where "." denotes elementwise multiplication and where $\tilde{\mathbf{H}}_{k}(z)$ and $\mathbf{S}_{k}$ are given by

$$
\begin{aligned}
\tilde{\mathbf{H}}_{k}(z)= & {\left[\begin{array}{cccc}
\tilde{H}_{k, 0}(z) & \tilde{H}_{k, 1}(z) & \cdots & \tilde{H}_{k, M-1}(z) \\
z^{-1} \tilde{H}_{k, M-1}(z) & \tilde{H}_{k, 0}(z) & \cdots & \tilde{H}_{k, M-2}(z) \\
z^{-1} \tilde{H}_{k, M-2}(z) & z^{-1} \tilde{H}_{k, M-1}(z) & \cdots & \tilde{H}_{k, M-3}(z) \\
\vdots & \vdots & \ddots & \vdots \\
z^{-1} \tilde{H}_{k, 1}(z) & z^{-1} \tilde{H}_{k, 2}(z) & \cdots & \tilde{H}_{k, 0}(z)
\end{array}\right] } \\
\mathbf{S}_{k}= & {\left[\begin{array}{cccc}
a_{k, 0} b_{k, 0} & a_{k, 1} b_{k, 0} & \cdots & a_{k, M-1} b_{k, 0} \\
a_{k, 0} b_{k, 1} & a_{k, 1} b_{k, 1} & \cdots & a_{k, M-1} b_{k, 1} \\
a_{k, 0} b_{k, 2} & a_{k, 1} b_{k, 2} & \cdots & a_{k, M-1} b_{k, 2} \\
\vdots & \vdots & \ddots & \vdots \\
a_{k, 0} b_{k, M-1} & a_{k, 1} b_{k, M-1} & \cdots & a_{k, M-1} b_{k, M-1}
\end{array}\right] . }
\end{aligned}
$$

Equation (11) can equivalently be written as

$$
\mathbf{S}_{k}=\mathbf{b}_{k}^{T} \mathbf{a}_{k}
$$

where

$$
\begin{aligned}
\mathbf{a}_{k} & =\left[\begin{array}{llll}
a_{k, 0} & a_{k, 1} & \cdots & a_{k, M-1}
\end{array}\right], \\
\mathbf{b}_{k} & =\left[\begin{array}{llll}
b_{k, 0} & b_{k, 1} & \cdots & b_{k, M-1}
\end{array}\right],
\end{aligned}
$$

and $T$ stands for transpose. Examples of the $\boldsymbol{S}_{k}$-matrices and of the $\mathbf{a}_{k}$ - and $\mathbf{b}_{k}$-vectors are provided for the TIM system in (26) and (25) in Example 1 in Section 4. Examples are also provided for the HM and FBDM systems in Examples 2 and 3 , respectively.

\subsubsection{Alias-free system}

With the system represented as above, it is known that it is alias-free, and thus time-invariant if and only if the matrix $\mathbf{P}(z)$ is pseudocirculant [19]. Under this condition, the output $z$-transform becomes

$$
Y(z)=H_{\mathrm{A}}(z) X(z),
$$

where

$$
\begin{aligned}
H_{\mathrm{A}}(z) & =z^{-M+1} \sum_{k=0}^{N-1} \sum_{i=0}^{M-1} s_{k}^{0, i} z^{-i} \tilde{H}_{k, i}\left(z^{M}\right) \\
& =\sum_{k=0}^{N-1} \sum_{i=0}^{M-1} s_{k}^{0, i} z^{-i} H_{k, i}\left(z^{M}\right)
\end{aligned}
$$

with $s_{k}^{0, i}$ denoting the elements on the first row of $\mathbf{S}_{k}$. This case corresponds to a Nyquist sampled ADC of which two

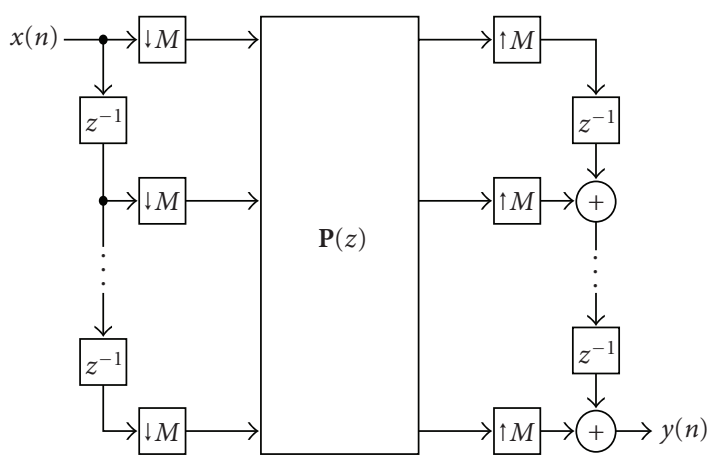

FIgURE 4: Equivalent representation of the system in Figure 1 based on the equivalences in Figure 2. $\mathbf{P}(z)$ is given by (8).

special cases are the TIM ADC [3, 12] and HM ADC in [6]. These systems are also described in the context of the multirate formulation in Examples 1 and 2 in Section 4.

Regarding $\tilde{\mathbf{H}}_{k}(z)$, it is seen in (10) that it is pseudocirculant for an arbitrary $\tilde{H}_{k}(z)$. It would thus be sufficient to make $S_{k}$ circulant for each channel $k$ in order to make each $\mathbf{P}_{k}(z)$ pseudocirculant and end up with a pseudocirculant $\mathbf{P}(z)$. Unfortunately, the set of circulant real-valued $\mathbf{S}_{k}$ achievable by the construction in (12) is seriously limited, because the rank of $\mathbf{S}_{k}$ is one. However, for purposes of error cancellation between channels it is beneficial to group the channels in sets where the matrices within each set sum to a circular matrix. The channel set $\{0,1, \ldots, N-1\}$ is thus partitioned into the sets $C_{0}, \ldots, C_{I-1}$, where each sum

$$
\sum_{k \in C_{i}} \mathbf{S}_{k}
$$

is a circulant matrix. It is assumed that the modulators and filters are identical for channels belonging to the same partition, $\mathbf{H}_{k}(z)=\mathbf{H}_{l}(z)$ whenever $k, l \in C_{i}$, and thus $\tilde{\mathbf{H}}_{k}(z)=$ $\widetilde{\mathbf{H}}_{l}(z)$. The matrix for partition $i$ is denoted $\widetilde{\mathbf{H}}_{0, i}(z)$. Sensitivity to channel mismatches are discussed further in Section 3.

\subsubsection{L-decimated alias-free system}

We say that a system is an $L$-decimated alias-free system if it is alias-free before decimation by a factor of $L$. A channel of such a system is shown in Figure 5(a). Obviously, the decimation can be performed before the modulation, as shown in Figure 5(b), if the index of the modulation sequence is scaled by a factor of $L$. Considering the equivalent system in Figure 5(c), it is apparent that the downsampling by $L$ can be moved to after the scalings by $b_{k, l}$ if the delay elements $z^{-1}$ are replaced by $L$-fold delay elements $z^{-L}$. The system may then be described as in Figure $5(\mathrm{~d})$, where $\mathbf{P}_{k}(z)$ is defined by (5). However, the outputs are taken from every $L$ th row of $\mathbf{P}_{k}(z)$, such that the first output $y_{k, L-1 \bmod M}(m)$ is taken from row $L$, the second output $y_{k, 2 L-1 \bmod M}(m)$ is taken from row $(2 L-1 \bmod M)+1$, and so on. It is thus apparent that only rows $\operatorname{gcd}(L, M) \cdot i, i=0,1,2, \ldots$, are used.

The $L$-decimated system corresponds to an oversampled ADC. The main observation that should be made is that the 


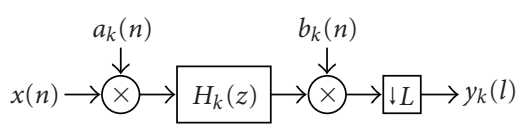

(a) Decimation at output

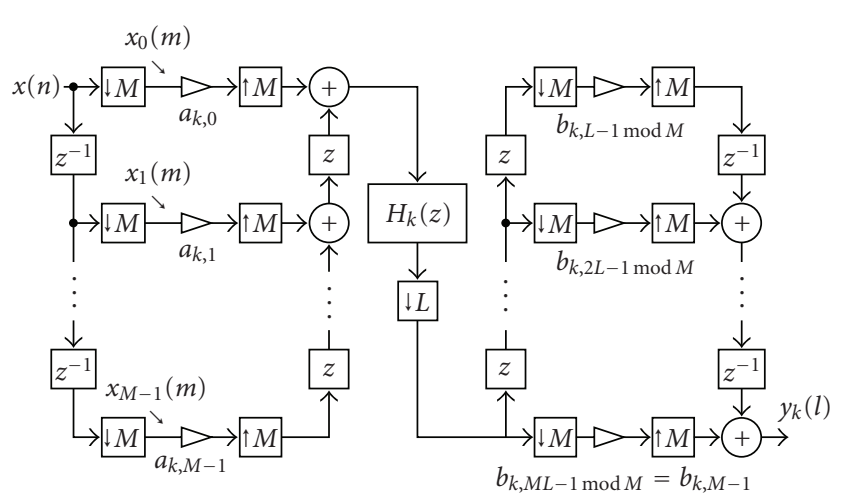

(c) Polyphase decomposition of input and output

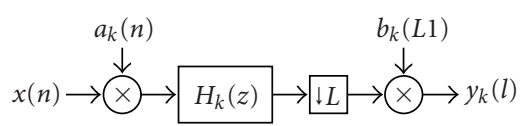

(b) Internal decimation

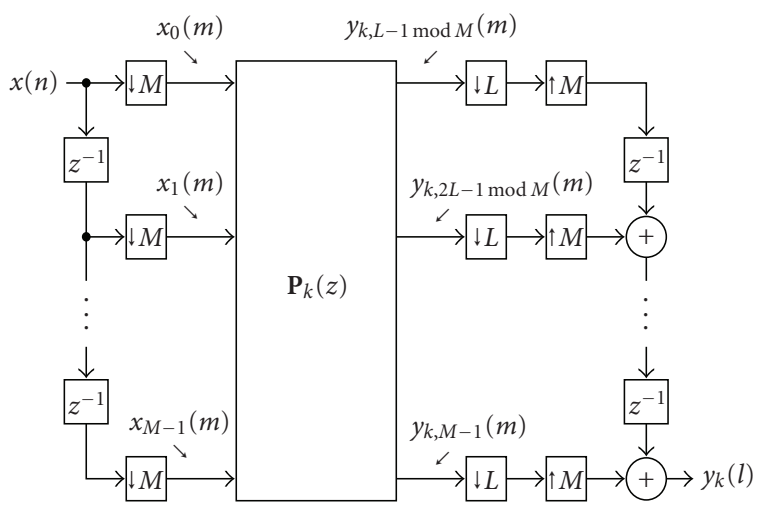

(d) Multirate formulation of a channel. $y_{k, L}(m)$ denotes the output pertaining to the $L$ th row of $\mathbf{P}_{k}(z)$

Figure 5: Channel model of $L$-decimated system.

$L$-decimated system may be described in the same way as the critically sampled system, but that relaxations may be allowed on the requirements of the modulation sequences. As only a subset of the rows of $\mathbf{P}(z)$ are used, the matrix needs only to be pseudocirculant on these rows. As in the critically sampled (nonoversampled) case, the channel set $\{0,1, \ldots, N-1\}$ is partitioned into sets $C_{0}, \ldots, C_{I-1}$ where the matrix $\sum_{k \in C_{i}} \boldsymbol{S}_{k}$ is circulant on the rows $\operatorname{gcd}(L, M) \cdot i$, $i=0,1,2, \ldots$, and $\widetilde{\mathbf{H}}_{k}(z)=\widetilde{\mathbf{H}}_{l}(z)=\widetilde{\mathbf{H}}_{0, i}(z)$ when $k, l \in C_{i}$. The oversampled Hadamard-modulated system in [7] belongs to this category of the formulation, and another example of a decimated system is given in Example 4 in Section 4.

\section{SENSITIVITY TO CHANNEL MISMATCHES}

In this section, the channel model used for the sensitivity analysis is explained. In the system shown in Figure 6, several nonidealities resulting from imperfect analog circuits have been included. Difficulties in realizing the exact values of the analog modulation sequence are modelled by an additive error term $\varepsilon_{k}(n)$. The error is assumed to be static, that is, it depends only on the value of $a_{k}(n)$, and is therefore a periodic sequence with the same periodicity as $a_{k}(n)$. The timevarying error $\varepsilon_{k}(n)$ may be a major concern when the modulation sequences contain nontrivial elements, that is, elements that are not $-1,0$, or 1 . The trivial elements may be realized without a multiplier by exchanging, grounding, or passing through the inputs to the modulator, and are for this reason particularly attractive on the analog side.

A channel-specific gain $\gamma_{k}$ is included in the sensitivity analysis, and analog imperfections in the modulator are modelled as the transfer function $\Delta H_{k}(z)$. The modulator nonidealities including channel gain and modulation sequence errors are analyzed separately in the context of the multirate formulation. In practice, there is also a channel off- set $\delta_{k}$ which is not suitable for analysis in this context, as it is signal independent. Channel offsets are commented in Section 3.3 below.

\subsection{Modulator nonidealities}

Assume that the ideal system is alias-free, that is, the ma$\operatorname{trix} \mathbf{P}(z)=\sum \mathbf{P}_{k}(z)$ is pseudocirculant. Due to analog circuit errors the transfer function of channel $k$ deviates from the ideal $H_{k}(z)$ to $\gamma_{k}\left(H_{k}(z)+\Delta H_{k}(z)\right)$, and $\tilde{H}_{k}(z)$ is replaced by $\hat{H}_{k}(z)=\gamma_{k}\left(H_{k}(z)+\Delta H_{k}(z)\right) z^{M-1}$. The transfer matrix for channel $k$ thus becomes $\hat{\mathbf{P}}_{k}(z)$ with elements

$$
\hat{P}_{k}^{j, i}(z)= \begin{cases}b_{k, j} \hat{H}_{k, i-j}(z) a_{k, i} & \text { for } i \geq j, \\ b_{k, j} z^{-1} \hat{H}_{k, i-j+M}(z) a_{k, i} & \text { for } i<j,\end{cases}
$$

where $\hat{H}_{k, p}(z)$ are the polyphase components of $\hat{H}_{k}(z)$. It is apparent that $\hat{\mathbf{P}}_{k}(z)$ is pseudocirculant whenever $\mathbf{P}_{k}(z)$ is. Thus a system where all the $\mathbf{S}_{k}$ matrices are circulant is completely insensitive to modulator mismatches.

In the general case, unfortunately, all $\boldsymbol{S}_{k}$ are not circulant and $\sum \hat{\mathbf{P}}_{k}(z)=\sum \mathbf{S}_{k} \cdot \hat{\mathbf{H}}_{k}(z)$ does not sum up to a pseudocirculant matrix as the matrices $\hat{\mathbf{H}}_{k}(z)$ are different between the channels. Partitioning the channel set into the sets $C_{i}$, as described in Section 2, and matching the modulators of channels belonging to the same partition $C_{i}$, that is, defining $\gamma_{k}=\gamma_{l}$ and $\Delta H_{k}(z)=\Delta H_{l}(z)$ when $k, l \in C_{i}$, allows $\hat{\mathbf{P}}(z)$ to be written

$$
\widehat{\mathbf{P}}(z)=\sum_{k=0}^{N-1} \mathbf{S}_{k} \cdot \hat{\mathbf{H}}_{k}(z)=\sum_{i=0}^{I-1} \hat{\mathbf{H}}_{0, i}(z) \cdot \sum_{k \in C_{i}} \mathbf{S}_{k},
$$


and it is apparent that each term in the outer sum is pseudocirculant, and thus that also $\mathbf{P}(z)$ is. Thus the system is alias-free and non-linear distortion is eliminated.

\subsection{Modulation sequence errors}

It is assumed that the ideal system is alias-free, that is, $\mathbf{P}(z)=$ $\sum \mathbf{P}_{k}(z)$ is pseudocirculant. Due to difficulties in realizing the analog modulation sequence, the signal is modulated in channel $k$ by the sequence $\hat{\mathbf{a}}_{k}=\mathbf{a}_{k}+\boldsymbol{\varepsilon}_{k}$ rather than the ideal sequence $\mathbf{a}_{k}$. We consider here different choices of the modulation sequences.

\subsubsection{Bilevel sequence for an insensitive channel}

Assume that an analog modulation sequence with two levels is used for an insensitive channel, that is, $\mathbf{S}_{k}=\mathbf{b}_{k}^{T} \mathbf{a}_{k}$ is a circular matrix. Examples of this type of channel include the first two channels of an HM system. Assuming that the sequence errors $\boldsymbol{\varepsilon}_{k}$ depend only on $\mathbf{a}_{\mathbf{k}}$, that is, $\varepsilon_{k, n_{1}}=\varepsilon_{k, n_{2}}$ when $a_{\left(k, n_{1}\right)}=a_{\left(k, n_{2}\right)}$, the modulation vector can be written $\hat{\mathbf{a}}_{k}=\alpha_{k} \mathbf{a}_{k}+\left[\beta_{k} \beta_{k} \cdots \beta_{k}\right]$ for some values of the scaling factor $\alpha_{k}$ and offset term $\beta_{k}$. The channel matrix $\hat{\mathbf{P}}_{k}(z)$ for the channel modulated with the sequence $\hat{\mathbf{a}}_{k}$ then becomes

$$
\begin{aligned}
\hat{\mathbf{P}}_{k}(z) & =\mathbf{b}_{k}^{T}\left(\alpha_{k} \mathbf{a}_{k}+\left[\begin{array}{llll}
\beta_{k} & \beta_{k} & \cdots & \beta_{k}
\end{array}\right]\right) \cdot \widetilde{\mathbf{H}}_{k}(z) \\
& =\alpha_{k} \mathbf{S}_{k} \cdot \widetilde{\mathbf{H}}_{k}(z)+\beta_{k} \mathbf{B}_{k} \widetilde{\mathbf{H}}_{k}(z),
\end{aligned}
$$

where $\mathbf{B}_{k}$ is a diagonal matrix consisting of the elements of $\mathbf{b}_{k}$. The first term is pseudocirculant, and thus the system is insensitive to modulation sequence scaling factors in channel $k$. The impact of the offset term $\beta_{k}$, that is, the second term, is explained under Section 3.2.4 below.

\subsubsection{Bilevel sequence for sensitive channels}

Consider one of the subsets $C_{i}$ in the partition of the channel set. The sum of the $S_{k}$-matrices corresponding to the channels in the set, $\sum_{k \in C_{i}} \mathbf{S}_{k}$, is a circulant matrix, whereas the constituent matrices are not. Examples of this type of channels are the TIM systems and the HM systems with more than two channels. As in the insensitive case, the modulation vectors are written $\hat{\mathbf{a}}_{k}=\alpha_{k} \mathbf{a}_{k}+\left[\beta_{k} \beta_{k} \cdots \beta_{k}\right]$, and the sum of the channel matrices for the channel subset becomes

$$
\begin{aligned}
& \sum_{k \in C_{i}} \hat{\mathbf{P}}_{k}(z)=\sum_{k \in C_{i}} \mathbf{b}_{k}^{T}\left(\alpha_{k} \mathbf{a}_{k}+\left[\begin{array}{llll}
\beta_{k} & \beta_{k} & \cdots & \beta_{k}
\end{array}\right]\right) \cdot \tilde{\mathbf{H}}_{k}(z) \\
& =\left(\tilde{\mathbf{H}}_{0, i}(z) \cdot \sum_{k \in C_{i}} \alpha_{k} \mathbf{S}_{k}\right)+\sum_{k \in C_{i}} \beta_{k} \mathbf{B}_{k} \tilde{\mathbf{H}}_{k}(z),
\end{aligned}
$$

where $\mathbf{B}_{k}$ is a diagonal matrix consisting of the elements of $\mathbf{b}_{k}$. The first sum is generally not a pseudocirculant matrix, and the channels are thus sensitive to sequence gain errors. If the gains are matched, denote $\alpha_{0, i}=\alpha_{k}=\alpha_{l}$ when $k, l \in C_{i}$, the channel matrix sum may be written

$$
\sum_{k \in C_{i}} \widehat{\mathbf{P}}_{k}(z)=\left(\alpha_{0, i} \widetilde{\mathbf{H}}_{0, i}(z) \cdot \sum_{k \in C_{i}} \mathbf{S}_{k}\right)+\sum_{k \in C_{i}} \beta_{k} \mathbf{B}_{k} \widetilde{\mathbf{H}}_{k}(z)
$$

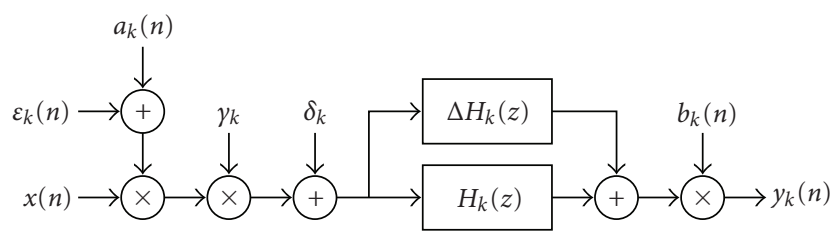

FIGURE 6: Channel model with nonideal analog circuits.

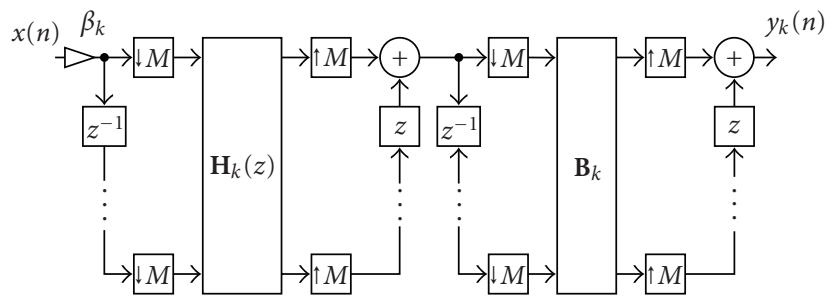

Figure 7: Model of errors in a parallel system pertaining to sequence offsets.

and it is seen that the first term is a pseudocirculant matrix, and the channel set is alias-free. Again, the impact of the offset term $\beta_{k}$ is explained under Section 3.2.4 below.

\subsubsection{Multilevel sequences}

If an insensitive channel is modulated with a multilevel sequence $\widehat{\mathbf{a}}_{k}=\mathbf{a}_{k}+\boldsymbol{\varepsilon}_{k}$, the channel matrix becomes

$$
\begin{aligned}
\hat{\mathbf{P}}_{k}(z) & =\mathbf{b}_{k}^{T}\left(\mathbf{a}_{k}+\boldsymbol{\varepsilon}_{k}\right) \cdot \widetilde{\mathbf{H}}_{k}(z) \\
& =\mathbf{S}_{k} \cdot \widetilde{\mathbf{H}}_{k}(z)+\mathbf{b}_{k}^{T} \boldsymbol{\varepsilon}_{k} \cdot \widetilde{\mathbf{H}}_{k}(z),
\end{aligned}
$$

which is pseudocirculant only if $\mathbf{b}_{k}^{T} \boldsymbol{\varepsilon}_{k}$ is a circulant matrix. Systems with multilevel analog modulation sequences are thus sensitive to level errors.

\subsubsection{Modulation sequence offset errors}

Consider here the modulation sequence offset errors introduced above under Sections 3.2.1 and 3.2.2. The channel matrix for a channel with a modulation sequence containing an offset error can be written as (19). Thus the error pertaining to the sequence offset is additive, and can be modelled as in Figure 7. The signal is thus first filtered through $H_{k}(z)$ and then aliased by the system $\mathbf{B}_{k}$, as $\mathbf{B}_{k}$ is not pseudocirculant unless the elements in the digital modulation sequence $\mathbf{b}_{k}$ are identical. However, as the signal is first filtered, only signal components in the passband of $H_{k}(z)$ will cause aliasing. If the signal contains no information in this band, aliasing will be completely suppressed. Typically the signal has a guard band either at the low-frequency or high-frequency region to allow transition bands of the filters, and the modulator can then be suitably chosen as either a lowpass type or highpass type, respectively. Errors pertaining to sequence offsets are demonstrated in Example 1 in Section 4. 


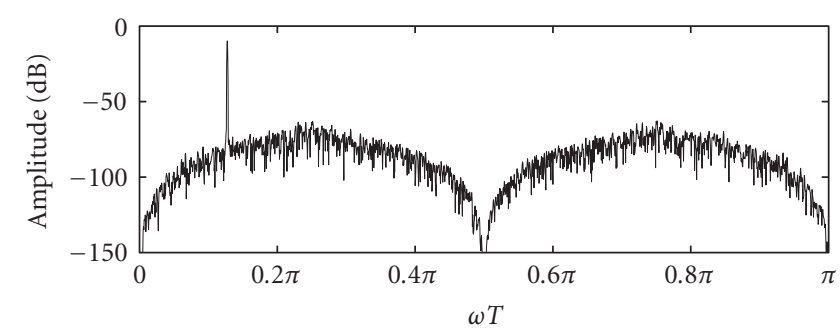

(a) Simulation using ideal system

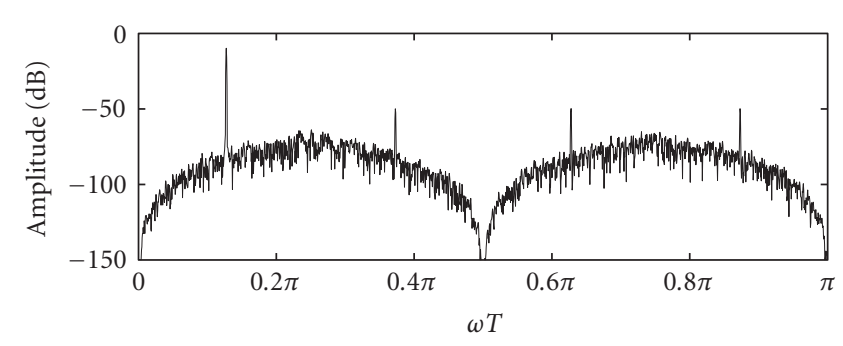

(c) Simulation with $1 \%$ offset error in one modulation sequence

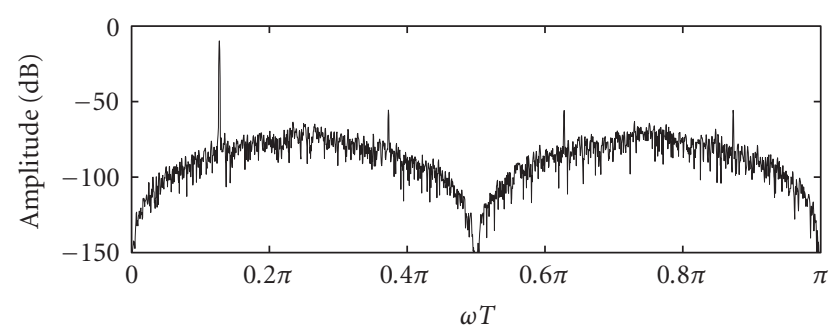

(b) Simulation with $2 \%$ gain mismatch in one channel

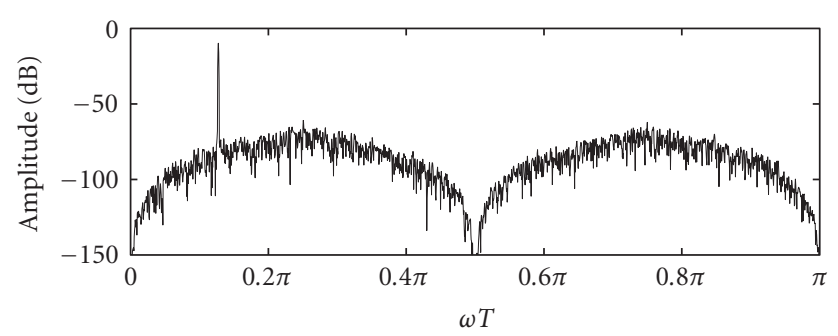

(d) Simulation with $1 \%$ offset error in one modulation sequence using highpass modulators instead of lowpass modulators

Figure 8: Sensitivity of TIM ADC in Example 1.

\subsection{Channel offset errors}

Channel offsets must be removed for each channel in order not to overload the $\Sigma \Delta$-modulator. Offsets affect the system in a nonlinear way and may not be analyzed using the multirate formulation. However, the problem has been well investigated and numerous solutions exist $[12,16,20]$.

\section{EXAMPLES}

In this section, examples of how the formulation can be used to analyze a system's sensitivity to channel mismatch errors are presented. Examples are provided for the TIM, HM, and FBDM ADCs. Also, an example is provided of how the formulation can be used to derive a new architecture that is insensitive to channel matching errors.

Example 1 (TIM ADC). Consider a TIM ADC [3, 4] with four channels. The samples are interleaved between the channels, each encompassing identical second-order lowpass modulators and decimation filters. Ideally, their $z$-domain transforms may be written

$$
H_{k}(z)=H(z)= \begin{cases}z^{-1}, & -\frac{\pi}{4} \leq \omega T \leq \frac{\pi}{4} \\ 0, & \text { otherwise. }\end{cases}
$$

All modulators are running at the input sampling rate, with their inputs grounded between consecutive samples. Thus the modulation sequences are

$$
\begin{aligned}
& a_{0}(n)=b_{0}(n)=1,0,0,0, \ldots, \\
& a_{1}(n)=b_{1}(n)=0,1,0,0, \ldots, \\
& a_{2}(n)=b_{2}(n)=0,0,1,0, \ldots, \\
& a_{3}(n)=b_{3}(n)=0,0,0,1, \ldots,
\end{aligned}
$$

all periodic with period $M=4$. The vectors $\mathbf{a}_{k}$ and $\mathbf{b}_{k}$ are as defined by (13):

$$
\begin{aligned}
& \mathbf{a}_{0}=\mathbf{b}_{3}=\left[\begin{array}{llll}
1 & 0 & 0 & 0
\end{array}\right] \\
& \mathbf{a}_{1}=\mathbf{b}_{0}=\left[\begin{array}{llll}
0 & 0 & 0 & 1
\end{array}\right] \\
& \mathbf{a}_{2}=\mathbf{b}_{1}=\left[\begin{array}{llll}
0 & 0 & 1 & 0
\end{array}\right] \\
& \mathbf{a}_{3}=\mathbf{b}_{2}=\left[\begin{array}{llll}
0 & 1 & 0 & 0
\end{array}\right]
\end{aligned}
$$

The matrices $\boldsymbol{S}_{k}$, defined by (12), then become

$$
\begin{aligned}
& \mathbf{S}_{0}=\mathbf{b}_{0}^{T} \mathbf{a}_{0}=\left[\begin{array}{llll}
0 & 0 & 0 & 0 \\
0 & 0 & 0 & 0 \\
0 & 0 & 0 & 0 \\
1 & 0 & 0 & 0
\end{array}\right], \\
& \mathbf{S}_{1}=\mathbf{b}_{1}^{T} \mathbf{a}_{1}=\left[\begin{array}{llll}
0 & 0 & 0 & 0 \\
0 & 0 & 0 & 0 \\
0 & 0 & 0 & 1 \\
0 & 0 & 0 & 0
\end{array}\right], \\
& \mathbf{S}_{2}=\mathbf{b}_{2}^{T} \mathbf{a}_{2}=\left[\begin{array}{llll}
0 & 0 & 0 & 0 \\
0 & 0 & 1 & 0 \\
0 & 0 & 0 & 0 \\
0 & 0 & 0 & 0
\end{array}\right], \\
& \mathbf{S}_{3}=\mathbf{b}_{3}^{T} \mathbf{a}_{3}=\left[\begin{array}{llll}
0 & 1 & 0 & 0 \\
0 & 0 & 0 & 0 \\
0 & 0 & 0 & 0 \\
0 & 0 & 0 & 0
\end{array}\right] .
\end{aligned}
$$

Because the sum of all $\mathbf{S}_{k}$-matrices is a circulant matrix, the system is alias-free and the transfer function for the system is given by $(15)$ as $H_{A}(z)=z^{-1} s_{3}^{0,1} H_{3,1}\left(z^{4}\right)=z^{-1}$ where $H_{3,1}(z)=1$ is the second polyphase component in the 
polyphase decomposition of $H(z)$. The transfer function is thus a simple delay, and the system will digitize the complete spectrum.

As none of the $\mathbf{S}_{k}$-matrices are circulant, and a circulant matrix can be formed only by summing all the matrices, the TIM ADC requires matching of all channels in order to eliminate aliasing. Thus we define $C_{0}=\{0,1,2,3\}$, according to the description in Section 2.1.1. The system has been simulated with modulator nonidealities and errors of bilevel sequences for sensitive channels, as described in Section 3. Figure 8 (a) shows the output spectrum for the ideal case with no mismatches between channels $\left(\gamma_{k}=1\right.$ for all $\left.k\right)$. Applying $2 \%$ gain mismatch for one of the channels $\left(\gamma_{0}=0.98\right.$, $\left.\gamma_{1}=\gamma_{2}=\gamma_{3}=1\right)$, the spectrum in Figure 8(b) results, where the aliasing components can be clearly seen. In Figure 8(c), the channel gains are set to one, and a $1 \%$ offset error has been added to the first modulation sequence $\left(\beta_{0}=0.01\right.$, $\left.\beta_{1}=\beta_{2}=\beta_{3}=0\right)$, which results in aliasing. In Figure $8(\mathrm{~d})$, high-pass modulators have been used instead, and the distortions disappear, as predicted by the analysis in Section 3.2.4.

Example 2 (HM ADC). Consider a nonoversampling HM ADC [6] with eight channels. In this case, every channel filter is an 8 th-band filter $\left(H_{k}(z)=H(z), k=0, \ldots, 7\right)$ and the modulation sequences $a_{k}(n)$ and $b_{k}(n)$ are

$$
\begin{aligned}
& a_{0}(n)=b_{0}(n)=1,1,1,1,1,1,1,1, \ldots, \\
& a_{1}(n)=b_{1}(n)=1,-1,1,-1,1,-1,1,-1, \ldots, \\
& a_{2}(n)=b_{2}(n)=1,1,-1,-1,1,1,-1,-1, \ldots, \\
& a_{3}(n)=b_{3}(n)=1,-1,-1,1,1,-1,-1,1, \ldots, \\
& a_{4}(n)=b_{4}(n)=1,1,1,1,-1,-1,-1,-1, \ldots, \\
& a_{5}(n)=b_{5}(n)=1,-1,1,-1,-1,1,-1,1, \ldots, \\
& a_{6}(n)=b_{6}(n)=1,1,-1,-1,-1,-1,1,1, \ldots, \\
& a_{7}(n)=b_{7}(n)=1,-1,-1,1,-1,1,1,-1, \ldots,
\end{aligned}
$$

The vectors $\mathbf{a}_{k}$ and $\mathbf{b}_{k}$ become

$$
\begin{aligned}
\mathbf{a}_{0}=\mathbf{b}_{0} & =\left[\begin{array}{llllllll}
1 & 1 & 1 & 1 & 1 & 1 & 1 & 1
\end{array}\right] \\
\mathbf{a}_{1}=-\mathbf{b}_{1} & =\left[\begin{array}{llllllll}
1 & -1 & 1 & -1 & 1 & -1 & 1 & -1
\end{array}\right] \\
\mathbf{a}_{2}=\mathbf{b}_{3} & =\left[\begin{array}{llllllll}
1 & -1 & -1 & 1 & 1 & -1 & -1 & 1
\end{array}\right] \\
\mathbf{a}_{3}=-\mathbf{b}_{2} & =\left[\begin{array}{lllllllll}
1 & 1 & -1 & -1 & 1 & 1 & -1 & -1
\end{array}\right] \\
\mathbf{a}_{4} & =\left[\begin{array}{llllllll}
1 & -1 & -1 & -1 & -1 & 1 & 1 & 1
\end{array}\right] \\
\mathbf{b}_{4} & =\left[\begin{array}{llllllll}
-1 & -1 & -1 & -1 & 1 & 1 & 1 & 1
\end{array}\right] \\
\mathbf{a}_{5} & =\left[\begin{array}{lllllllll}
1 & 1 & -1 & 1 & -1 & -1 & 1 & -1
\end{array}\right] \\
\mathbf{b}_{5} & =\left[\begin{array}{llllllll}
1 & -1 & 1 & -1 & -1 & 1 & -1 & 1
\end{array}\right] \\
\mathbf{a}_{6} & =\left[\begin{array}{lllllllll}
1 & 1 & 1 & -1 & -1 & -1 & -1 & 1
\end{array}\right] \\
\mathbf{b}_{6} & =\left[\begin{array}{llllllll}
1 & 1 & -1 & -1 & -1 & -1 & 1 & 1
\end{array}\right] \\
\mathbf{a}_{7} & =\left[\begin{array}{lllllllll}
1 & -1 & 1 & 1 & -1 & 1 & -1 & -1
\end{array}\right] \\
\mathbf{b}_{7} & =\left[\begin{array}{llllllll}
-1 & 1 & 1 & -1 & 1 & -1 & -1 & 1
\end{array}\right]
\end{aligned}
$$

With $\mathbf{S}_{k}=\mathbf{b}_{k}^{T} \mathbf{a}_{k}$, the following matrices can be computed:

$$
\begin{aligned}
\mathbf{S}_{0} & =1 \\
\mathbf{S}_{1} & =\left[\begin{array}{cccccccc}
-1 & 1 & -1 & 1 & -1 & 1 & -1 & 1 \\
1 & -1 & 1 & -1 & 1 & -1 & 1 & -1 \\
-1 & 1 & -1 & 1 & -1 & 1 & -1 & 1 \\
1 & -1 & 1 & -1 & 1 & -1 & 1 & -1 \\
-1 & 1 & -1 & 1 & -1 & 1 & -1 & 1 \\
1 & -1 & 1 & -1 & 1 & -1 & 1 & -1 \\
-1 & 1 & -1 & 1 & -1 & 1 & -1 & 1 \\
1 & -1 & 1 & -1 & 1 & -1 & 1 & -1
\end{array}\right], \\
\mathbf{S}_{2}+\mathbf{S}_{3} & =\left[\begin{array}{cccccccc}
0 & 2 & 0 & -2 & 0 & 2 & 0 & -2 \\
-2 & 0 & 2 & 0 & -2 & 0 & 2 & 0 \\
0 & -2 & 0 & 2 & 0 & -2 & 0 & 2 \\
2 & 0 & -2 & 0 & 2 & 0 & -2 & 0 \\
0 & 2 & 0 & -2 & 0 & 2 & 0 & -2 \\
-2 & 0 & 2 & 0 & -2 & 0 & 2 & 0 \\
0 & -2 & 0 & 2 & 0 & -2 & 0 & 2 \\
2 & 0 & -2 & 0 & 2 & 0 & -2 & 0
\end{array}\right], \\
\mathbf{S}_{4}+\mathbf{S}_{5}+\mathbf{S}_{6}+\mathbf{S}_{7} & =\left[\begin{array}{cccccccc}
0 & 4 & 0 & 0 & 0 & -4 & 0 & 0 \\
0 & 0 & 4 & 0 & 0 & 0 & -4 & 0 \\
0 & 0 & 0 & 4 & 0 & 0 & 0 & -4 \\
-4 & 0 & 0 & 0 & 4 & 0 & 0 & 0 \\
0 & -4 & 0 & 0 & 0 & 4 & 0 & 0 \\
0 & 0 & -4 & 0 & 0 & 0 & 4 & 0 \\
0 & 0 & 0 & -4 & 0 & 0 & 0 & 4 \\
4 & 0 & 0 & 0 & -4 & 0 & 0 & 0
\end{array}\right] .
\end{aligned}
$$

It is seen that $\mathbf{S}_{0}$ and $\mathbf{S}_{1}$ are circulant matrices. Also, $\mathbf{S}_{2}+\mathbf{S}_{3}$ is circulant. Further, the remaining matrices sum to a circulant matrix $\mathbf{S}_{4}+\mathbf{S}_{5}+\mathbf{S}_{6}+\mathbf{S}_{7}$, whereas no smaller subset does. Thus, in order to eliminate aliasing, the channels are partitioned into the sets $C_{0}=\{0\}, C_{1}=\{1\}, C_{2}=\{2,3\}$, and $C_{3}=\{4,5,6,7\}$. The HM ADC thus contains both insensitive channels 0 and 1 , and sensitive channels $2, \ldots, 7$.

Using the model of the ideal system, the spectrum of the output signal is as shown in Figure 9(a). Figure 9(b) shows the output spectrum for the system with $1 \%$ random gain mismatch $\left(\gamma_{k} \in[0.99,1.01]\right)$, where the aliasing distortions are readily seen. Matching the gains of the $C_{2}$-channels to each other (setting $\gamma_{2}=\gamma_{3}$ ) and the gains of the $C_{3}$-channels to each other (setting $\gamma_{4}=\gamma_{5}=\gamma_{6}=\gamma_{7}$ ), the spectrum in Figure 9(c) results, and the distortions disappear. Although the HM ADC is less sensitive than the TIM ADC, the matching requirements for eight-channel systems and above are still severe. Another limitation is that the reduced sensitivity seemingly requires a number of channels that are a power of two. For Hadamard matrices of other orders, extensive searches by the authors have not yielded solutions with simplified matching requirements.

Example 3 (FBDM ADC). For the FBDM ADC, the input signal is applied unmodulated to $N$ modulators converting different frequency bands. Consider as an example a fourchannel system consisting of a lowpass channel, a highpass 


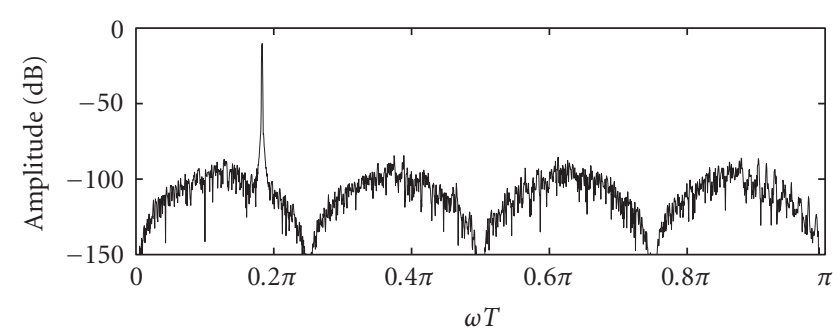

(a) Simulation using ideal model

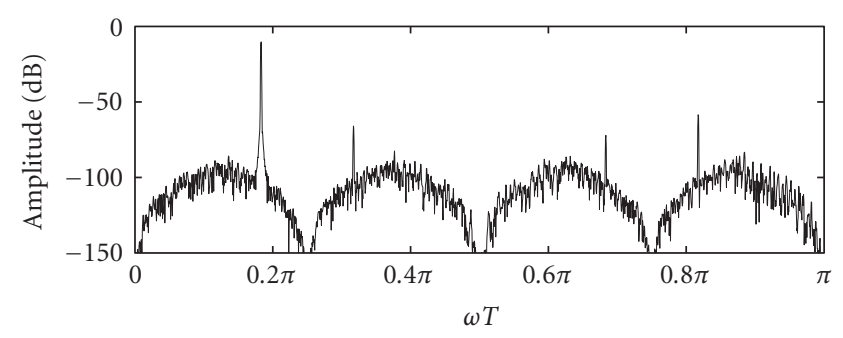

(b) Simulation using 1\% channel gain mismatch

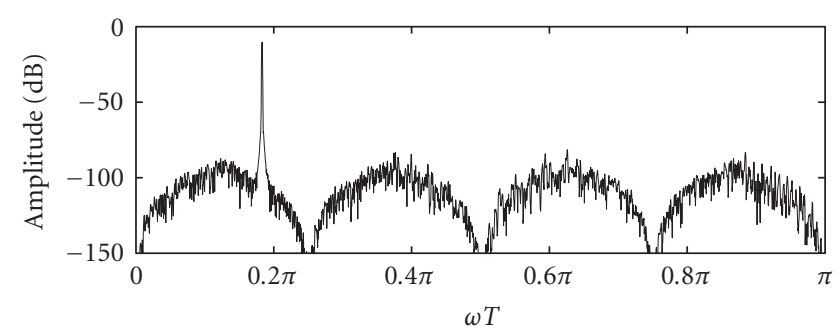

(c) Simulation using gain matching of sensitive channels

FIgure 9: Sensitivity of TIM ADC in Example 2.

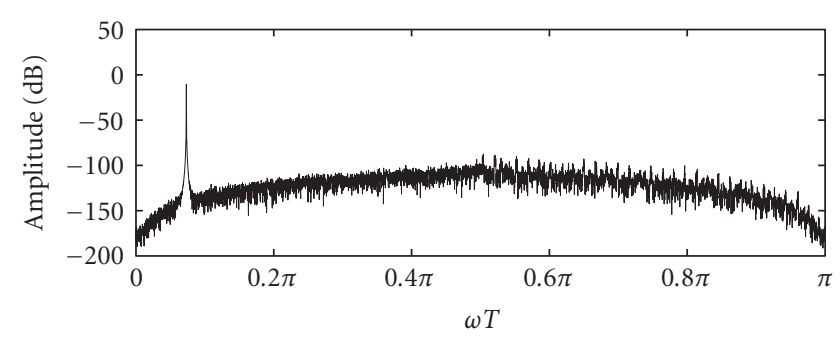

FIgURE 10: Sensitivity of new scheme in Example 4. Simulation using $10 \%$ channel gain mismatch.

channel, and two bandpass channels centered at $3 \pi / 8$ and $5 \pi / 8$.

As the signal is not modulated,

$$
\mathbf{a}_{k}=\mathbf{b}_{k}=\left[\begin{array}{llll}
1 & 1 & 1 & 1
\end{array}\right]
$$

for all $k$, and

$$
\mathbf{S}_{k}=\left[\begin{array}{llll}
1 & 1 & 1 & 1 \\
1 & 1 & 1 & 1 \\
1 & 1 & 1 & 1 \\
1 & 1 & 1 & 1
\end{array}\right]
$$

for all $k$. As each $\boldsymbol{S}_{k}$-matrix is circulant, the system is insensitive to channel mismatches. Further, modulation sequence errors are irrelevant in this case, as the signal is not modulated. The FBDM ADC is thus highly resistant to mismatches. Its obvious drawback, however, is the need to use bandpass modulators which are more expensive in hardware.

Example 4 (generation of new scheme). This example demonstrates that the formulation can also be used to devise new schemes, although a general method is not presented. A three-channel parallel system using lowpass modulators is designed. The signal is assumed to be in the frequency band $-\pi / 4<\omega T<\pi / 4$, and the ADC is thus an oversampled system and is described according to Section 2.1.2 with $L=4$ and $M=8$.

Using complex modulation sequences, three bands of width $\pi / 4$ centered at $-\pi / 4,0$, and $\pi / 4$ can be translated to baseband and converted with a lowpass ADC. These modulation sequences are $a_{0}(n)=1, a_{1}(n)=\exp (j \pi n / 4), a_{2}(n)=$ $\exp (-j \pi n / 4)$, and $b_{k}(n)=a_{k}^{*}(n)$. Summing the resultant $\boldsymbol{S}_{k^{-}}$ matrices yields

$$
\begin{aligned}
& \sum \mathbf{S}_{k} \\
& =1+\left[\begin{array}{cccccccc}
\sqrt{2} & 2 & \sqrt{2} & 0 & -\sqrt{2} & -2 & -\sqrt{2} & 0 \\
0 & \sqrt{2} & 2 & \sqrt{2} & 0 & -\sqrt{2} & -2 & -\sqrt{2} \\
-\sqrt{2} & 0 & \sqrt{2} & 2 & \sqrt{2} & 0 & -\sqrt{2} & -2 \\
-2 & -\sqrt{2} & 0 & \sqrt{2} & 2 & \sqrt{2} & 0 & -\sqrt{2} \\
-\sqrt{2} & -2 & -\sqrt{2} & 0 & \sqrt{2} & 2 & \sqrt{2} & 0 \\
0 & -\sqrt{2} & -2 & -\sqrt{2} & 0 & \sqrt{2} & 2 & \sqrt{2} \\
\sqrt{2} & 0 & -\sqrt{2} & -2 & -\sqrt{2} & 0 & \sqrt{2} & 2 \\
2 & \sqrt{2} & 0 & -\sqrt{2} & -2 & -\sqrt{2} & 0 & \sqrt{2}
\end{array}\right] .
\end{aligned}
$$

Unfortunately, using complex modulation sequences is not practical. However, as the modulators and filters are identical for all channels $\left(H_{k}(z)=H(z)\right.$ for all $\left.k\right)$, any other choice of modulation sequences resulting in the same matrix will perform the same function. Moreover, for a decimated system, relaxations may be allowed on the new modulation sequences. In this case, with decimation by four, it is sufficient to find replacing modulation sequences $\mathbf{a}_{k}^{\prime}$ and $\mathbf{b}_{k}^{\prime}$ such that the sum of the resulting $\boldsymbol{S}_{k}^{\prime}$-matrices equals $\sum \boldsymbol{S}_{k}$ on rows 4 and 8 , as $\operatorname{gcd}(L, M)=4$. One such choice of modulation sequences is

$$
\begin{aligned}
& \mathbf{a}_{0}^{\prime}=\left[\begin{array}{llllllll}
1 & 1 & 1 & 1 & 1 & 1 & 1 & 1
\end{array}\right], \\
& \mathbf{a}_{1}^{\prime}=\left[\begin{array}{llllllll}
1 & 1 & 0 & -1 & -1 & -1 & 0 & 1
\end{array}\right], \\
& \mathbf{a}_{2}^{\prime}=\left[\begin{array}{llllllll}
1 & 0 & 0 & 0 & -1 & 0 & 0 & 0
\end{array}\right], \\
& \mathbf{b}_{0}^{\prime}=\left[\begin{array}{llllllll}
0 & 0 & 0 & 1 & 0 & 0 & 0 & 1
\end{array}\right] \text {, } \\
& \mathbf{b}_{1}^{\prime}=\left[\begin{array}{llllllll}
0 & 0 & 0 & -\sqrt{2} & 0 & 0 & 0 & \sqrt{2}
\end{array}\right] \text {, } \\
& \mathbf{b}_{2}^{\prime}=\left[\begin{array}{llllllll}
0 & 0 & 0 & (\sqrt{2}-2) & 0 & 0 & 0 & (2-\sqrt{2})
\end{array}\right] \text {. }
\end{aligned}
$$

The analog modulation sequences $\mathbf{a}_{k}^{\prime}$ can easily be implemented by switching or grounding the inputs to the 
modulators, whereas the nontrivial multiplications in $\mathbf{b}_{k}^{\prime}$ can be implemented with high precision digitally. Note that

$$
\begin{aligned}
& \sum \mathbf{b}_{k}^{\prime T} \mathbf{a}_{k}^{\prime} \\
& =1+\left[\begin{array}{cccccccc}
0 & 0 & 0 & 0 & 0 & 0 & 0 & 0 \\
0 & 0 & 0 & 0 & 0 & 0 & 0 & 0 \\
0 & 0 & 0 & 0 & 0 & 0 & 0 & 0 \\
-2 & -\sqrt{2} & 0 & \sqrt{2} & 2 & \sqrt{2} & 0 & -\sqrt{2} \\
0 & 0 & 0 & 0 & 0 & 0 & 0 & 0 \\
0 & 0 & 0 & 0 & 0 & 0 & 0 & 0 \\
0 & 0 & 0 & 0 & 0 & 0 & 0 & 0 \\
2 & \sqrt{2} & 0 & -\sqrt{2} & -2 & -\sqrt{2} & 0 & \sqrt{2}
\end{array}\right],
\end{aligned}
$$

which is equal to $\sum \mathbf{S}_{k}$ in (33) on rows 4 and 8 . Note also that the $\mathbf{S}_{k}^{\prime}$-matrices, given on rows 4 and 8 by

$$
\begin{aligned}
& {\left[\begin{array}{l}
\mathbf{b}_{0,3}^{\prime} \\
\mathbf{b}_{0,7}^{\prime}
\end{array}\right] \mathbf{a}_{0}^{\prime}=\left[\begin{array}{llllllll}
1 & 1 & 1 & 1 & 1 & 1 & 1 & 1 \\
1 & 1 & 1 & 1 & 1 & 1 & 1 & 1
\end{array}\right],} \\
& {\left[\begin{array}{l}
\mathbf{b}_{1,3}^{\prime} \\
\mathbf{b}_{1,7}^{\prime}
\end{array}\right] \mathbf{a}_{1}^{\prime}=\left[\begin{array}{cccccccc}
-\sqrt{2} & -\sqrt{2} & 0 & \sqrt{2} & \sqrt{2} & \sqrt{2} & 0 & -\sqrt{2} \\
\sqrt{2} & \sqrt{2} & 0 & -\sqrt{2} & -\sqrt{2} & -\sqrt{2} & 0 & \sqrt{2}
\end{array}\right],} \\
& {\left[\begin{array}{l}
\mathbf{b}_{2,3}^{\prime} \\
\mathbf{b}_{2,7}^{\prime}
\end{array}\right] \mathbf{a}_{2}^{\prime}=\left[\begin{array}{llllllll}
(\sqrt{2}-2) & 0 & 0 & 0 & (2-\sqrt{2}) & 0 & 0 & 0 \\
(2-\sqrt{2}) & 0 & 0 & 0 & (\sqrt{2}-2) & 0 & 0 & 0
\end{array}\right],}
\end{aligned}
$$

are circulant on these rows, and thus the system is insensitive to channel mismatches. This is demonstrated in Figure 10, where the channel gain mismatch is $10 \%$ and no aliasing results. However, as three levels are used in the analog modulation sequences $\mathbf{a}_{1}^{\prime}$ and $\mathbf{a}_{2}^{\prime}$, the system is sensitive to mismatches in the modulation sequences of these channels, as described in Section 3.

\section{NOISE MODEL OF SYSTEM}

The primary purpose of this paper is to investigate the signal transfer characteristics of the parallel $\Sigma \Delta$-system. However, the system's noise properties are also affected by the choice of modulation sequences, and therefore a simple noise analysis is included.

A noise model of the parallel $\Sigma \Delta$-system can be depicted as in Figure 11. The quantization noise $q_{k}(n)$ of channel $k$ is filtered through the noise transfer function $\operatorname{NTF}_{k}(z)$ and filter $G_{k}(z)$. The filtered noise is then modulated by the sequence $b_{k}(n)$. The channels are summed to form the output $y(n)$.

In order to determine the statistical properties of the output $y(n)$, channel $k$ is modeled as in Figure 12. Denoting the spectral density of the quantization noise of channel $k$ by $R_{Q_{k}}\left(e^{j \omega}\right)$, the spectral densities of the polyphase components $y_{k, m}$ of the channel output can be written

$$
R_{y_{k, m}}\left(e^{j \omega}\right)=b_{k, m}^{2} \sum_{l=0}^{M-1}\left|G_{k, l}\left(e^{j \omega}\right)\right|^{2} R_{Q_{k}}\left(e^{j \omega}\right),
$$

where $G_{k, l}(z)$ are the polyphase components of the cascaded system $\operatorname{NTF}_{k}(z) G_{k}(z)$. It is seen that the noise power is scaled

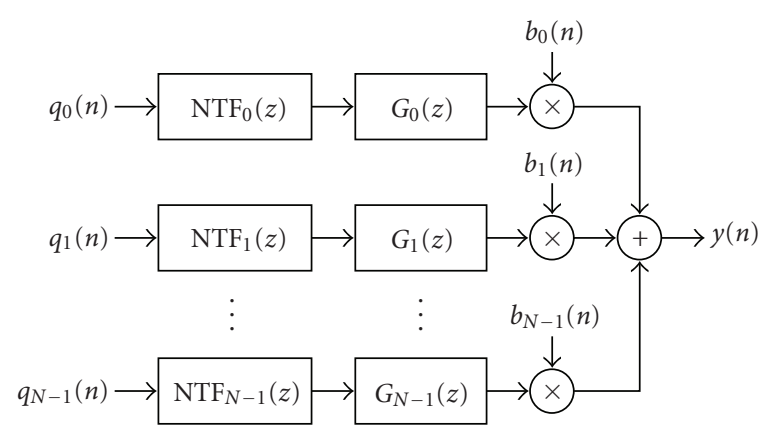

FIGURE 11: Noise model of parallel system.

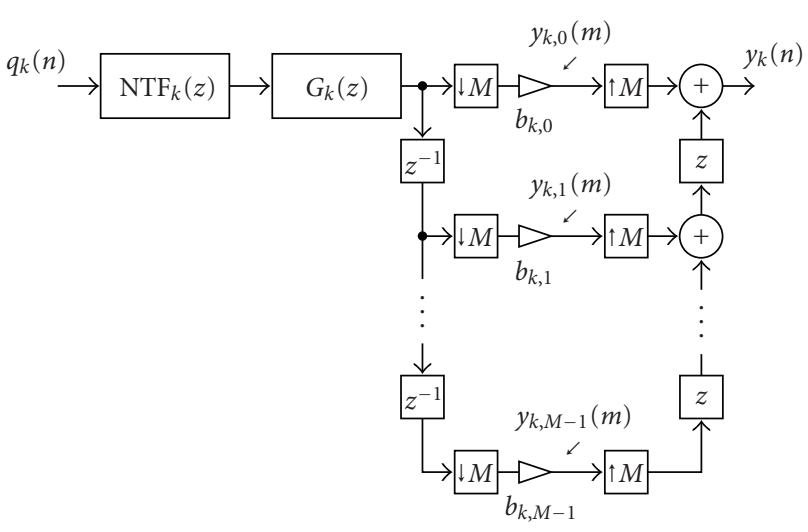

FIGURE 12: Noise model of chann $k$.

by the factor $b_{k, m}^{2}$, and it is thus of interest to keep the amplitudes of the modulation sequences low on the digital side. For example, in Example 4, alternative choices of $\mathbf{a}_{1}$ and $\mathbf{b}_{2}$ would have been

$$
\begin{aligned}
& \mathbf{a}_{1}=\left[\begin{array}{llllllll}
0 & 1 & 0 & -1 & 0 & -1 & 0 & 1
\end{array}\right], \\
& \mathbf{b}_{2}=\left[\begin{array}{llllllll}
0 & 0 & 0 & -2 & 0 & 0 & 0 & 2
\end{array}\right] .
\end{aligned}
$$

However, in this case the noise power is larger. This shows that the smaller magnitudes of the digital modulation sequences, as in (36), is preferable from a noise perspective.

\section{CONCLUSION}

In this paper, a new general formulation of analog-to-digital converters using parallel $\Sigma \Delta$-modulators was introduced. The TIM-, HM-, and FBDM ADCs have been described as special cases of this formulation, and it was shown how the model can be used to analyze the sensitivity to channel matching errors for a parallel system. Both Nyquist-rate and oversampled systems have been considered, and it was shown that an oversampled system may have a reduced sensitivity to mismatches, which may be determined using the formulation. The usefulness of the formulation is not limited to analysis of existing schemes, but also for the derivation of new ones, which was exemplified. 


\section{ACKNOWLEDGEMENT}

The work is financially supported by the Swedish Research Council.

\section{REFERENCES}

[1] L. J. Breems, R. Rutten, and G. Wetzker, "A cascaded continuous-time $\Sigma \Delta$ modulator with $67-\mathrm{dB}$ dynamic range in $10-\mathrm{MHz}$ bandwidth," IEEE Journal of Solid-State Circuits, vol. 39, no. 12, pp. 2152-2160, 2004.

[2] N. Yaghini and D. Johns, "A $43 \mathrm{~mW} C \mathrm{CT}$ complex $\Delta \Sigma \mathrm{ADC}$ with $23 \mathrm{MHz}$ of signal bandwidth and 68.8dB SNDR," in International Solid-State Circuits Conference, Digest of Technical Papers, pp. 502-503, February 2005.

[3] A. Eshraghi and T. S. Fiez, "A time-interleaved parallel $\Delta \Sigma$ A/D converter," IEEE Transactions on Circuits and Systems II, vol. 50, no. 3, pp. 118-129, 2003.

[4] A. Eshraghi and T. S. Fiez, "A comparative analysis of parallel delta-sigma ADC architectures," IEEE Transactions on Circuits and Systems I, vol. 51, no. 3, pp. 450-458, 2004.

[5] E. King, F. Aram, T. Fiez, and I. Galton, "Parallel delta-sigma A/D conversion," in Proceedings of the Custom Integrated Circuits Conference, pp. 503-506, 1994.

[6] I. Galton and H. T. Jensen, "Delta-sigma modulator based A/D conversion without oversampling," IEEE Transactions on Circuits and Systems II, vol. 42, no. 12, pp. 773-784, 1995.

[7] I. Galton and H. T. Jensen, "Oversampling parallel delta-sigma modulator A/D conversion," IEEE Transactions on Circuits and Systems II, vol. 43, no. 12, pp. 801-810, 1996.

[8] S. K. Kong and W. H. Ku, "Frequency domain analysis of $\Pi \Delta \Sigma$ $\mathrm{ADC}$ and its application to combining subband decomposition and $\Pi \Delta \Sigma$ ADC," in Proceedings of the Midwest Symposium on Circuits and Systems, vol. 1, pp. 226-229, August 1997.

[9] R. F. Cormier, T. L. Sculley, and R. H. Bamberger, "Combining subband decomposition and sigma delta modulation for wideband A/D conversion," in Proceedings of the IEEE International Symposium on Circuits and Systems, vol. 5, pp. 357-360, 1994.

[10] G. Ding, C. Dehollain, M. Declercq, and K. Azadet, "Frequency-interleaving technique for high-speed A/D conversion," in Proceedings of the IEEE International Symposium on Circuits and Systems, vol. 1, pp. I857-I860, May 2003.

[11] R. Khoini-Poorfard and D. A. Johns, "Time-interleaved oversampling convertors," Electronics Letters, vol. 29, no. 19, pp. 1673-1674, 1993.

[12] R. Khoini-Poorfard, L. B. Lim, and D. A. Johns, "Timeinterleaved oversampling A/D converters: theory and practice," IEEE Transactions on Circuits and Systems II, vol. 44, no. 8, pp. 634-645, 1997.

[13] M. Kozak, M. Karaman, and I. Kale, "Efficient architectures for time-interleaved oversampling delta-sigma converters," IEEE Transactions on Circuits and Systems II, vol. 47, no. 8, pp. 802810, 2000.

[14] F. Colodro, A. Torralba, and M. Laguna, "Time-interleaved multirate sigma-delta modulators," in Proceedings of the IEEE International Symposium on Circuits and Systems, vol. 6, pp. 5581-5584, Kobe, Japan, May 2005.

[15] R. Khoini-Poorfard and D. A. Johns, "Mismatch effects in time-interleaved oversampling converters," in Proceedings of the IEEE International Symposium on Circuits and Systems, vol. 5, pp. 429-432, 1994.
[16] R. D. Batten, A. Eshraghi, and T. S. Fiez, "Calibration of parallel $\Delta \Sigma$ ADCs," IEEE Transactions on Circuits and Systems II, vol. 49, no. 6, pp. 390-399, 2002.

[17] V. T. Nguyen, P. Loumeau, and J.-F. Naviner, "Analysis of timeinterleaved delta-sigma analog to digital converter," in Proceedings of the IEEE Vehicular Technology Conference, vol. 4, September 2002.

[18] A. Blad, H. Johansson, and P. Löwenborg, "A general formulation of analog-to-digital converters using parallel sigma-delta modulators and modulation sequences," in Proceedings of the Asia-Pacific Conference on Circuits and Systems, pp. 4-7, Singapore, December 2006.

[19] P. P. Vaidyanathan, Multirate Systems and Filter Banks, Prentice-Hall, Eaglewood Cliffs, NJ, USA, 1993.

[20] S. R. Norsworthy, R. Schreier, and G. C. Temes, Eds., DeltaSigma Data Converters: Theory, Design, and Simulation, WileyIEEE Press, New York, NY, USA, 1996. 